\title{
Cognition and native-language grammar: The organizational role of adjective-noun word order in information representation
}

\author{
Elise J. Percy and Steven J. Sherman \\ Indiana University, Bloomington, Indiana \\ LeONEl Garcia-Marques \\ University of Lisbon, Lisbon, Portugal \\ ANDRÉ MATA \\ Instituto Superior de Ciências do Trabalho e da Empresa, Lisbon, Portugal \\ AND \\ Teresa Garcia-Marques \\ Instituto Superior de Psicologia Aplicada, Lisbon, Portugal
}

\begin{abstract}
In the present research, we investigated the influence of native-language adjective-noun word order on category accessibility for nouns and adjectives by comparing Portuguese speakers (in whose language nouns precede adjectives) with English speakers (in whose language adjectives precede nouns). In two studies, we presented participants with different numbers of verbal or pictorial stimuli, and subsequently they answered questions about noun- and adjective-conditioned frequencies. The results demonstrated a primacy effect of nativelanguage word order. Specifically, although both populations showed a speed advantage for noun-conditioned questions, this tendency was significantly stronger for Portuguese than for American participants. We discuss the important role of native-language syntax rules for the categorization and representation of information.
\end{abstract}

Decades of inquiry have gone into investigating the notion that language shapes human experience, on the premise that the world is highly complicated and that language assists in making it palatable and understandable. In the present work, we address a related, but more specific, proposition: that the various ways in which languages grammatically organize experience lead to different ways of cognitively organizing experience.

Whorf's (1956) linguistic relativity principle is the idea that differences across languages can lead to corresponding differences in cognition. Although early studies (e.g., Heider \& Olivier, 1972) were unsupportive of Whorf, more recently linguistic relativity has taken on a new face in a new generation of empirical work that has successfully demonstrated the important role of language in cognitive processes. Characteristic of this recent work is the proposition that, even if language does not determine thought, there still exists much room for the influence of language. These are commonly referred to as the strong and weak forms of the Whorfian hypothesis, respectively (see Boroditsky, 2001). Strong linguistic relativity purports that language severely limits thought, such that subtleties of experience not labeled by one's language are literally imperceptible.
Its weak counterpart implies crosslinguistic differences in cognitive tendency, rather than potentiality. It is this weak form that is the focus of much recent work.

For example, Boroditsky, Schmidt, and Phillips (2003) investigated the effects of gendered articles (through which certain objects are arbitrarily given either feminine or masculine designations) on object perception. They presented speakers of Spanish and German with objects of opposing genders between the two languages. Participants' descriptions were congruent with each object's grammatical gender in their native language. For example, key was described as stereotypically masculine (e.g., "heavy, jagged, metal") by Germans, but as feminine (e.g., "golden, intricate, little") by Spaniards. Similar crosslinguistic differences have been demonstrated for perceptions of causality as a function of causal language rules (Fausey \& Boroditsky, 2008), the ability to remember and match arrays of objects as a function of available counting words (Frank, Everett, Fedorenko, \& Gibson, 2008), stereotypic judgments as a function of available stereotype labels (Hoffman, Lau, \& Johnson, 1986), and perceptions of colors as a function of available color words (Roberson, Davies, \& Davidoff, 2000). 


\section{The Present Studies:}

\section{General Rationale and Approach}

Unlike many earlier investigations, the present study examines not what happens when speakers of a language lack particular labels, but how speakers of different languages with different syntax rules behave in situations in which multiple labels apply. For example, a red shirt is both a red thing and a shirt. A person organizing a pile of clothes might sort it into the "red" pile or into the "shirt" pile, depending on whether the task was doing laundry or putting clothes into drawers. Thus, individuals can use different rules of organization to arrange the same objects in various ways.

Of interest in the present work is the association between the ease with which one can categorize an item in particular ways and the manner in which it is habitually described. Regardless of the context, English speakers would refer to the aforementioned item as "the red shirt." On the other hand, Portuguese speakers generally use a noun-adjective word order and say (when translated literally) "[The shirt red]."

Such crosslinguistic differences in adjective-noun word order are well documented (e.g., Dryer, 2007; Greenberg, 1963). To quantify this difference for English and Portuguese, we coded the order of nouns and their modifying adjectives in five randomly selected chapters from Collodi's (1883) The Adventures of Pinocchio, translated into English (1986) and Portuguese (1999). In the English translation, adjectives preceded the nouns they modified $77 \%$ of the time, whereas in the Portuguese translation, nouns preceded adjectives $67 \%$ of the time. Thus, English and Portuguese clearly differ in their employment of adjective-noun order.

Importantly, the relationship between this syntactic feature of language and issues of cognitive representation has not yet been examined systematically. These differences in the way in which objects are habitually spoken about may lead to differences in the way these items are represented, but what particular representational differences might one expect?

\section{Evidence for Primacy Effects}

There is reason to believe that the order in which descriptive words are encountered is important to the way in which the target object is categorized. For example, Asch (1946) presented participants with lists of traits for an impression-formation task and found that targets were rated more positively when the positive traits appeared first than when they appeared later (see also Tavassoli \& Lee, 2004).

Such work, when applied to word-order conventions, anticipates a language-based primacy effect, such that the element appearing first in the native-language word order (adjective or noun) takes relative precedence in the categorization of objects. Although recency effects are theoretically plausible and have been demonstrated in such domains as memory and persuasion, primacy dominates over recency in the context of categorization (Duffy \& Crawford, 2008), the most relevant domain for the present research.

\section{Hypotheses}

The present research was designed in light of (1) the growing body of evidence demonstrating the effects of language over cognitive processes, (2) the well-known differences in adjective-noun word order between languages, and (3) the documented existence of primacy effects in information representation. The general hypothesis guiding the present work is that differences in native-language adjective-noun word order are associated with differences in the accessibility of objects by their noun and adjective categories. Considering our red shirt example, English speakers might be expected to associate the object more closely with the category "red things" than would Portuguese speakers. Conversely, Portuguese speakers would be expected to associate it more closely with the category "shirts" than would English speakers.

According to Lambert and Paivio (1956; see also Paivio, 1963), even in English, nouns constitute conceptual "pegs" from which their modifiers are "hung." Thus, noun categories may generally be more dominant than adjectives are in categorization. However, the degree of this privileged status for nouns is proposed here to vary as a function of the order in which adjectives and nouns appear in one's language. Specifically, such noun dominance should be stronger for Portuguese than for English speakers.

\section{Measurement of Category Accessibility: Conditional-Frequency Judgments}

Relative accessibility of categories can be measured in various ways. The chosen method for the present work was to employ judgments of conditional frequency. These involve assessing the frequency of one event given the cooccurrence of another. Sherman, McMullen, and Gavanski (1992) investigated responses to conditional judgments of probability (e.g., "Of 100 randomly chosen men, how many prefer blue to brown?"). They concluded that the ease of these judgments depends on the individual's ability to first access the "sample space" of the conditional category (i.e., men) from which to then judge the probability of the target category (i.e., those who prefer blue to brown). Some categories have a more accessible sample space than do others. That is, some sets of information (e.g., men) come to mind more quickly than do others (e.g., people who prefer blue to brown) and thus are easier categories upon which to conditionalize. In the present studies, both latency and accuracy were measured for conditional-frequency judgments.

\section{STUDY 1}

\section{Predictions for Response Latency}

Language syntax conventions were expected to be associated with differences in category accessibility. More specifically, adjective-conditioned questions (which involve first accessing an adjective category) were anticipated to be answered more slowly than noun-conditioned questions (which involve first accessing a noun category), but this tendency was expected to be stronger for Portuguese participants than for American participants. In other words, the speed cost of answering adjective-conditioned 
questions was hypothesized to be attenuated for English speakers. This effect would be demonstrated by an interaction between languages (Portuguese vs. English) and question conditionality (noun vs. adjective) on response latency.

\section{Predictions for Response Accuracy}

Although the predictions for response latency are clear, predictions for response accuracy are not. One possibility is that, although participants may take additional time if they have difficulty accessing a category, they eventually access the appropriate category and use it to make judgments. Thus, both populations may have the same categories in memory but take different amounts of time to access them, such that differences in latency would be expected, whereas differences in accuracy would not. A second possibility is that, for "difficult" categories, individuals may adopt different strategies for answering the question or simply never gain access to an accurate "sample space." As a result, they might be both slower and less accurate.

American and Portuguese participants viewed a set of 81 short phrases (e.g., "the capable chef") in their own languages. Participants were then asked to provide conditional-frequency estimates for particular phrases. These judgments were either noun conditioned (e.g., "Of all the journalists described, how many were understanding?") or adjective conditioned (e.g., "Of all the understanding people described, how many were journalists?").

\section{Method}

Participants. A group of 186 English-speaking students from Indiana University constituted the American sample; 3 were eliminated due to self-reported fluency in a noun-first language, $(n=$ 183). One hundred ten Portuguese-speaking students from the Instituto Superior de Psicologia Aplicada constituted the Portuguese sample. Because most Portuguese college students have a great deal of exposure to English, Portuguese participants were included, regardless of experience with adjective-first languages.

Stimuli. In order to include a variety of object types in the experiment, participants viewed one of three types of stimuli: text describing colors and clothing (e.g., "the purple dress"), mixed objects and attributes (e.g., "the costly painting"), or traits and occupations (e.g., "the meek clerk"). Each stimulus set consisted of nine nouns and nine adjectives. The lists of English nouns and adjectives were matched in word length and frequency, using the Kučera and Francis (1967) corpus. The Portuguese lists were matched in word length, but not in word frequency. ${ }^{1}$ To maintain consistency across the translations, all words and phrases were devised under the supervision of coauthors fluent in both languages. The nouns and adjectives were randomly paired to form 27 simple, sensible phrases.

Procedure. We varied the number of times that a particular phrase was presented (one, three, or five times, counterbalanced), and the combined frequencies for all phrases always totaled 81 presentations, which were shown in a randomized order. The same phrases and frequencies were presented to participants in their native languages (thus conforming to each language's adjective-noun word-order conventions).

This experiment employed a 2 (between subjects: language) $\times 2$ (within subjects: question conditionality) design.

Before presentation of the stimuli, participants were told to read the items carefully, because they would be asked about what they had seen. Each phrase remained on the computer screen for 3,000 msec, with a $1,500-\mathrm{msec}$ pause between phrases. After the last phrase was presented, participants were presented 60 questions (30 adjec- tive conditioned, 30 noun conditioned) of conditional frequency on the screen, consisting of noun and adjective conditionality questions about the stimuli they encountered (e.g., "Of all the paintings, how many were costly?"; "Of all the costly things, how many were paintings?", respectively). Participants were instructed to answer as quickly and accurately as possible by typing a response to each of the questions in a free-response window and hitting the "Enter" key to submit the response. In order to avoid the consecutive appearance of complementary questions (i.e., questions in which conditionality was reversed), a blocking procedure was employed. The questions were presented in two main blocks of 29 questions separated from one another by a 2-question buffer block. Adjective- and noun-conditioned questions were distributed evenly across these blocks. Among the 60 questions, 4 (1 in the first main block, 1 in the second main block, and 2 constituting the buffer block) were included for which the correct response was 0 (i.e., the words had appeared, but never together in the same phrase). The order of the two main blocks was counterbalanced, with the buffer block always appearing between the two main blocks. The order of questions was randomized within each block.

Accuracy and latency of the responses to the conditionalfrequency questions were recorded.

\section{Results}

A response and its accompanying latency were eliminated if any of the following were true: (1) Latency was less than $500 \mathrm{msec}$, (2) latency was more than $2 S D$ s above the mean latency for that sample, or (3) the response was above 10 (at most, the correct answer was 5 , so responses exceeding 10 were very extreme). These cutoffs led to the elimination of fewer than $6 \%$ of all responses.

Results indicated that participants correctly differentiated the presentation frequencies (zero, one, three, or five) of particular phrases, with higher estimates for phrases with higher presentation frequencies $[F(3,876)=$ $308.340, p<.001]$. Planned contrasts revealed significant differences between all pairs (all $p \mathrm{~s}<.001$ ), indicating that participants were sensitive to the presentation frequencies of the stimuli.

The primary analyses of interest tested the relationship between native language and type of question conditionality. ${ }^{2}$ Noun-defined categories were expected to be generally more accessible than were adjective-defined categories, but the degree of this difference was expected to vary as a function of the grammar of the participant's native language. Specifically, the speed cost for adjectiveconditioned judgments was expected to be attenuated for the American sample.

Response latency. Most importantly, a language $\times$ question conditionality ANOVA revealed an interactive effect in the expected pattern on response latency $[F(1,291)=3.889, p=.05]$, demonstrating that the relative speed of accessing noun and adjective categories varied in accordance with native-language adjective-noun

Table 1

Study 1 Response Latency (in Milliseconds)

\begin{tabular}{lcccrr}
\hline & \multicolumn{3}{c}{$\begin{array}{c}\text { Portuguese } \\
\text { Participants }\end{array}$} & & \multicolumn{2}{c}{$\begin{array}{c}\text { American } \\
\text { Participants }\end{array}$} \\
\cline { 2 - 3 } \cline { 5 - 6 } Conditionality & $M$ & $S D$ & & $M$ & $S D$ \\
\hline Adjective & $4,529.64$ & 866.38 & & $4,235.68$ & $1,012.37$ \\
Noun & $4,093.12$ & 823.73 & & $3,914.43$ & 943.22 \\
\hline
\end{tabular}


word-order primacy effects (see Table 1 for cell means). In support of Lambert and Paivio's (1956) claim that nouns act as "conceptual pegs," we obtained a significant main effect of question conditionality, such that participants responded more quickly to noun-conditioned questions than to adjective-conditioned questions $[F(1,291)=168.078$, $p<.001]$. However, the degree of the speed cost for adjective-conditioned judgments was greater among the Portuguese sample $\left(\eta_{\mathrm{p}}^{2}=.404\right)$ than among the American sample $\left(\eta_{\mathrm{p}}^{2}=.335\right)$.

An uninteresting main effect of language was also obtained: Response latencies were shorter for American than for Portuguese participants $[F(1,291)=4.751, p=$ $.03]$, likely due to the greater experience of the American sample with speeded computerized tasks.

Response accuracy. ${ }^{3}$ Inaccuracy of responses was calculated by using the absolute differences of responses from the correct answers to the conditional-frequency questions. A language $\times$ question conditionality ANOVA showed no significant interaction or main effect of conditionality (both $F \mathrm{~s}<1$ ). Only a main effect of language was obtained: Portuguese participants tended to be less accurate overall $[F(1,291)=9.251, p=.003]$.

\section{Discussion}

Study 1 demonstrated the effect of the hypothesized language $\times$ question conditionality interaction on response latency. The stimuli could be categorized by noun (e.g., "shirt") or by the modifying adjective (e.g., "red"). The response latency interaction obtained supports the hypothesis that the relative ease of categorizing items by the relevant noun as compared with the effort required in categorizing them by the relevant adjective is related to the word order of one's native language. With regard to response accuracy, only a main effect of language was found, implying that the interaction of interest is related to how quickly noun and adjective categories can be accessed, but not to how accurate those judgments are after that category has been accessed.

\section{STUDY 2}

In Study 1, the phrases that were employed followed natural language order. Thus, the most recent language experience of participants was in the adjective-noun (for Americans) or noun-adjective (for Portuguese) order. In order to control for presentation word order, the stimuli for Study 2 were all pictorial - various shapes of different colors. This procedure also allowed an assessment of whether the differences in Study 1 would generalize to the representation of extralinguistic (visual) information.

\section{Method}

The design of Study 2 was identical to that in Study 1, except that, instead of verbal phrases, a single type of visual stimuli (colored shapes) was employed. The study again employed a 2 (language: English vs. Portuguese) $\times 2$ (question conditionality: noun vs. adjective) design.

Participants. A group of 67 native English speakers from Indiana University participated in exchange for course credit; 5 re-
Table 2

Study 2 Response Latency (in Milliseconds)

\begin{tabular}{lccccc}
\hline \multirow{2}{*}{ Conditionality } & \multicolumn{2}{c}{$\begin{array}{c}\text { Portuguese } \\
\text { Participants }\end{array}$} & & \multicolumn{2}{c}{$\begin{array}{c}\text { American } \\
\text { Participants }\end{array}$} \\
\cline { 2 - 3 } \cline { 5 - 6 } \cline { 5 - 6 } Adjective & $M$ & $S D$ & & $M$ & $S D$ \\
Noun & $4,641.77$ & 828.34 & & $3,475.83$ & 901.36 \\
\hline
\end{tabular}

ported fluency in a language using a noun-adjective word order and were excluded, leaving 62 English speakers. The Portuguese sample comprised 161 Portuguese-speaking students from the University of Lisbon.

Stimuli. The stimuli (colors and shapes) were devised by coupling nine colors with nine shapes to create 27 colored shapes. Only the most simple and common colors and shapes for both populations were used (e.g., red, blue, circle, square). As a result of this prioritization, in both Portuguese and English the adjectives (colors) were more common in the language than the nouns (shapes). ${ }^{4}$

Procedure. The experimental procedure was largely the same as that in Study 1. As was the case in that study, each stimulus was presented one, three, or five times, with the number of stimulus presentations always totaling to 81 . The same images were presented to American and Portuguese participants. Afterward, they answered 60 noun- and adjective-conditioned questions about what they had seen.

Instead of the free-response format used in Study 1, responses to the frequency questions were limited to a particular range of numeric responses. For each question, participants were instructed to "respond by pressing a numeric key (0-9) on the keyboard."

\section{Results}

As was the case in Study 1, a response and its accompanying latency were eliminated if any of the following were true: (1) Latency was less than $500 \mathrm{msec}$, or (2) latency was more than $2 S D$ s above the mean latency for that sample. These cutoffs led to the elimination of fewer than $6 \%$ of all responses.

Participants again successfully differentiated presentation frequencies (zero, one, three, or five) of particular images, giving higher estimates for higher presentation frequencies $[F(3,666)=222.639, p<.001]$, with significant differences between all pairs (all $p s<.001$ ).

Response latency. Most important to the hypothesis, a language $\times$ question conditionality ANOVA revealed the hypothesized interactive effect in the expected pattern on response latency $[F(1,221)=4.389, p=.037$; see Table 2 for cell means]. As in Study 1, participants responded more quickly to noun-conditioned questions than to adjective-conditioned questions $[F(1,221)=40.915, p<$ $.001]$. However, the speed cost for adjective-conditioned questions was greater for the Portuguese sample $\left(\eta_{\mathrm{p}}^{2}=\right.$ .264) than for the American sample $\left(\eta_{\mathrm{p}}^{2}=.139\right)$.

Additionally, a main effect of language showed that American participants had shorter response latencies than Portuguese participants did $[F(1,221)=80.697, p<$ $.001]$.

Response accuracy. As before, inaccuracy was calculated by using the absolute differences of responses from the correct answers to the conditional-frequency questions. A language $\times$ question conditionality ANOVA showed no significant interaction or main effects on response accuracy. 


\section{Discussion}

Study 2 provided additional support for the importance of native-language adjective-noun order for cognition. Even without a verbal description of the objects, the pictorial stimuli were represented in a way that reflected the order of adjectives and nouns within the language. Thus, for a blue circle, the blueness was relatively more accessible for the American than for the Portuguese participants. Results of this study demonstrate that such languagespecific effects can generalize beyond verbally presented information to an extralinguistic (pictorial) context.

\section{GENERAL DISCUSSION}

In two studies, the evidence supported the hypothesis of syntactic primacy effects in categorization. For both visual and verbal stimuli, Portuguese participants (noun first) showed a greater speed advantage for accessing noun categories than did American participants (adjective first).

The present work provides additional evidence to support the claims of weak linguistic relativity-specifically, that language effects may not exert themselves over cognitive capabilities, but rather over natural tendencies. This research shows that, although participants were capable of categorizing information by both adjective and noun, the order in which such elements appear in the grammar of one's native language is associated with differences in the relative ease of engaging in such categorizations.

Additional work to further develop these findings could take a variety of forms. One intriguing domain is that of similarity judgment (see Niedenthal, Halberstadt, \& InnesKer, 1999). Speakers of noun-first languages would be expected to weight nouns more heavily in assessing similarity than would speakers of adjective-first languages. That is, there would be a difference in the conceptual coherence of noun versus adjective categories. Such tendencies could have important perceptual and cognitive ramifications. Another particularly relevant domain is that of social impression formation. It is plausible that speakers of noun-first languages form stereotypes, subtypes, and impressions relatively more often on the basis of noun forms (e.g., social categories), whereas speakers of adjective-first languages may rely more on adjectival elements (e.g., traits) in developing and maintaining social knowledge structures. Thus, in making additional inferences about an honest teacher, speakers of adjective-first languages would rely relatively more on the teacher's honesty, whereas speakers of nounfirst languages would rely more on the teacher's occupational designation.

An important follow-up to this work will be research that isolates the mechanisms by which this word-order phenomenon occurs. For example, it may be that such effects will obtain only for contexts in which individuals implicitly can name the objects that they encounter (i.e., encoding the words "red triangle" upon seeing such a colored shape). Manipulations that could interfere with such naming, such as articulatory suppression, could provide further insight into the processes and boundaries of these kinds of effects.
It should be noted that, to fully elucidate the nature of the phenomena under study, future work must investigate the operation of such crosslinguistic differences in a richer context that more closely approximates real-life situations and that incorporates additional languages and populations. Such investigations would also provide helpful insight into the natural and spontaneous operation of these processes on a larger scale.

In sum, the present work demonstrates an important but heretofore uninvestigated influence on cognition: that of native-language syntax. Our results suggest that such crosslinguistic differences in word order may be quite consequential. In fact, such differences may constitute a new direction for the study of language and cognition, while adding a new voice to the chorus of recent research demonstrating that the language one speaks indeed bears important significance for the particular manner in which one thinks.

\section{AUTHOR NOTE}

Send correspondence to E. J. Percy, Department of Psychological and Brain Sciences, Indiana University, 1101 E. 10th St., Bloomington, IN 47405 (e-mail: ejpercy@indiana.edu).

\section{REFERENCES}

Asch, S. E. (1946). Forming impressions of personality. Journal of Abnormal \& Social Psychology, 41, 258-290.

Boroditsky, L. (2001). Does language shape thought? Mandarin and English speakers' conceptions of time. Cognitive Psychology, 43, $1-22$.

Boroditsky, L., Schmidt, L. A., \& Phillips, W. (2003). Sex, syntax, and semantics. In D. Gentner \& S. Goldin-Meadow (Eds.), Language in mind: Advances in the study of language and thought (pp. 61-79). Cambridge, MA: MIT Press.

Collodi, C. (1986). The adventures of Pinocchio (N. J. Perella, Trans.). Berkeley: University of California Press. (Original work published 1883)

Collodi, C. (1999). As aventuras de Pinóquio (J. C. Barreiros, Trans.). Lisbon: Caminho. (Original work published 1883)

DrYer, M. S. (2007). Word order. In T. Shopen (Ed.), Language typology and syntactic description: Vol. 1. Clause structure (pp. 61-131). Cambridge: Cambridge University Press.

Duffy, S., \& CRAWford, L. E. (2008). Primacy or recency effects in forming inductive categories. Memory \& Cognition, 36, 567-577.

FAusey, C. M., \& Boroditsky, L. (2008, July). English and Spanish speakers remember causal agents differently. In Proceedings of the 30th Annual Meeting of the Cognitive Science Society. Washington, DC.

Frank, M. C., Everett, D. L., Fedorenko, E., \& Gibson, E. (2008). Number as a cognitive technology: Evidence from Pirahã language and cognition. Cognition, 108, 819-824.

GreenberG, J. H. (1963). Some universals of grammar with particular reference to the order of meaningful elements. In J. H. Greenberg (Ed.), Universals of language (pp. 73-113). Cambridge, MA: MIT Press.

Heider, E. R., \& Olivier, D. C. (1972). The structure of the color space in naming and memory for two languages. Cognitive Psychology, 3, 337-354.

Hoffman, C., Lau, I., \& Johnson, D. R. (1986). The linguistic relativity of person cognition: An English-Chinese comparison. Journal of Personality \& Social Psychology, 51, 1097-1105.

Kučera, H., \& Francis, W. N. (1967). Computational analysis of present-day American English. Providence, RI: Brown University Press.

Lambert, W. E., \& Paivio, A. (1956). The influence of noun-adjective order on learning. Canadian Journal of Psychology, 10, 9-12.

Niedenthal, P. M., Halberstadt, J. B., \& Innes-Ker, A. H. (1999). 
Emotional response categorization. Psychological Review, 106, 337361.

Paivio, A. (1963). Learning of adjective-noun paired associates as a function of adjective-noun word order and noun abstractness. Canadian Journal of Psychology, 17, 370-379.

Roberson, D., Davies, I., \& Davidoff, J. (2000). Color categories are not universal: Replications and new evidence from a stone-age culture. Journal of Experimental Psychology: General, 129, 369-398.

Sherman, S. J., McMullen, M. N., \& Gavanski, I. (1992). Natural sample spaces and the inversion of conditional judgments. Journal of Experimental Social Psychology, 28, 401-421.

TaVassoli, N. T., \& LEe, Y. H. (2004). The effect of attribute order on judgment in Chinese and English. Journal of Experimental Psychology: Applied, 10, 258-266.

WHORF, B. L. (1956). Language, thought, and reality: Selected writings of Benjamin Lee Whorf (J. B. Carroll, Ed.). Cambridge, MA: MIT Press.

\section{NOTES}

1. Although word frequency for Portuguese stimuli was not controlled, analyses that statistically controlled for the factor of word frequency in individual stimulus items showed that this factor did not moderate the interaction of interest.

2. Analyses including the factor of stimulus set showed that it did not have a main effect and did not moderate the interaction of interest.

3. Analyses on underestimation/overestimation (the raw difference between responses and correct answers) similarly yielded null results for the interaction of interest.

4. Again, analyses employing word frequency as a covariate did not show that this factor moderated the interaction of interest.

(Manuscript received July 28, 2008;

revision accepted for publication August 10, 2009.) 